\title{
A qualitative study of interprofessional learning related to electronic health record (EHR) medication reconciliation within a social knowledge networking (SKN) system
}

This article was published in the following Dove Press journal:

Journal of Healthcare Leadership

\author{
Pavani Rangachari ${ }^{1}$ \\ Kevin C Dellsperger ${ }^{2}$ \\ R Karl Rethemeyer ${ }^{3}$ \\ 'Department of Interdisciplinary Health \\ Sciences, College of Allied Health \\ Sciences, Augusta University, Augusta, \\ GA 30912, USA; ${ }^{2}$ Cardiovascular \\ Division, AU Health, Department of \\ Medicine, Medical College of Georgia, \\ Augusta University, Augusta, GA, 309/2, \\ USA; ${ }^{3}$ Rockefeller College of Public \\ Affairs and Policy, University at Albany, \\ State University of New York, Albany, \\ NY, 12222, USA
}

Background: Similar to issues faced in health systems across the USA, AU Health faced a scenario of low physician engagement in and limited use of its Electronic Health Record (EHR) Medication Reconciliation (MedRec) technology, which translated to high rates of medication discrepancies and low accuracy of the patient's active medication list, during transitions of care. In fall 2016, a 2-year research grant was secured to pilot a Social Knowledge Networking (SKN) system on "EHR MedRec" to enable AU Health to progress from "limited use" of EHR MedRec technology to "meaningful use."

Purpose: The aims of this study were to 1) examine dynamics of interprofessional knowledge exchange and learning related to EHR MedRec on the SKN system and 2) explore associations between "SKN Use" and "Meaningful Use (MU) of EHR MedRec," with the latter being assessed in terms of adherence to best practices in EHR MedRec.

Methods: Over a 1-year period, 50 SKN Users (practitioners from inpatient and outpatient medicine settings), participated in discussing issues related to EHR MedRec, moderated by five SKN Moderators (senior administrators). Qualitative analysis was used to understand dynamics of interprofessional knowledge exchange and descriptive analysis was used to examine trends in two measures of MU of EHR MedRec, identified for the study.

Results: Interprofessional knowledge exchanges related to EHR MedRec on the SKN system, progressed from "problem statements" to "problem-solving statements" to "IT system education" to "best-practice assertions" to "culture change assertions" to "collective learning (aha) moments" to lay a foundation for practice change. These interprofessional learning dynamics were associated with distinct improvement trends in both measures of MU of EHR MedRec technology.

Conclusion: Results suggest that an SKN system could be a valuable tool in enabling MU of EHR MedRec technology. The study helps identify strategies for the creation of "learning health systems," to enable successful change implementation in healthcare organizations.

Keywords: interprofessional learning, qualitative analysis, electronic health records, medication reconciliation, meaningful use, change implementation

\section{Introduction}

Healthcare reform efforts in the United States have increasingly focused on improving patient safety and quality during transitions of care, when patients are known to be most vulnerable to medical errors. Transitions of care (from outpatient to
Correspondence: Pavani Rangachari Department of Interdisciplinary Health Sciences, College of Allied Health

Sciences, Augusta University, 987 St. Sebastian Way, Augusta, GA 30912, USA Tel +I 9642702214

Fax +I 7067216067

Email prangachari@augusta.edu 
inpatient, and back to outpatient settings), are commonly experienced by patients with chronic conditions (eg, heart disease, diabetes, and stroke). Medication errors, in particular, are common at hospital admission and discharge, and are known to contribute significantly to both adverse patient outcomes and higher costs associated with transitions of care. According to the Institute of Medicine, medication errors injure over 1.5 million people and cost billions of dollars each year. Additionally, the average hospitalized patient is known to experience at least one medication error per day. ${ }^{1}$ The risk of medication errors is heightened during care transitions, because providers, and sometimes, patients, do not have access to accurate active medication lists. This can result in the inadvertent addition, omission or duplication of medications, resulting in "unintended discrepancies" between what patients should be prescribed, and what they are actually prescribed. To help prevent medication discrepancies and errors during transitions of care, patient safety advocates have long promoted the use of medication reconciliation. ${ }^{1,2}$

Medication Reconciliation (MedRec) is a formal process for creating a complete and accurate list of a patient's current medications during transitions of care. The process of MedRec consists of several key steps: (1) develop a list of the patient's current medications; (2) develop a list of the medications to be prescribed; (3) compare the medications on the two lists; (4) make clinical decisions to update the medication list, based on the comparison; and (5) communicate the updated medication list to both the patient/family and the next providers of care. The goal of MedRec is to reduce medication discrepancies and errors during care transitions, and provide an accurate active medication list to patients/families and their next providers of care across the continuum, to promote patient safety and quality of care. ${ }^{1,2}$

MedRec has been a part of the Joint Commission hospital accreditation requirements since 2005, and with the introduction of the Health Information Technology for Economic and Clinical Health (HITECH) Act in 2009, it has become part of the Electronic Health Record (EHR) "Meaningful Use" requirements. Meaningful Use (MU) of EHR MedRec technology refers to effective use of the "EHR MedRec" system by providers, to complete the MedRec process (outlined above), to reduce medication discrepancies and promote medication list accuracy, during transitions of care. ${ }^{3,4}$

Despite the regulatory impetus towards MU of EHR MedRec however, hospital adherence has been found to lag due to low physician engagement in EHR MedRec, stemming in part, from lack of professional consensus about which physician (eg, hospital vs community physician) is responsible for managing a patient's medication list, and the value of MedRec as a clinical tool for promoting patient safety. ${ }^{5-8}$ Additionally, within the hospital context, the assignment of MedRec responsibilities among provider subgroups-multiple physicians, nurses, and pharmacists - is often unclear, leading to inefficiency and potential for error. ${ }^{9,10}$

Consistent with these findings, several recent studies have found that although hospital EHR vendors have been enhancing MedRec functionality over time, numerous hospitals still use partially paper-based processes during care transitions, which translates to limited use of the MedRec functionality on the EHR. ${ }^{11-13}$ In other words, there is "limited use" of EHR MedRec technology, in hospitals and health systems across the US, as opposed to "meaningful use."14,15 This paper discusses a health system's initiative, to pilot a Social Knowledge Networking (SKN) system on EHR MedRec, to enable it to progress from "limited use" to "meaningful use" of EHR MedRec technology. The rationale is that an SKN system would enable knowledge exchange on practice issues related to EHR MedRec, across diverse provider subgroups and care settings, which, in turn, is expected to increase provider engagement in addressing those issues, promote interprofessional learning of best-practices, and provide a foundation for practice change or improvement (ie, Meaningful Use of EHR MedRec technology).

\section{Problem of interest}

Based in Augusta, GA, USA, Augusta University's Health System, AU Health, is a healthcare network offering comprehensive primary, specialty and subspecialty care in the region. Facilities include a 478-bed AU Medical Center, more than 80 outpatient practice sites, a Critical Care Center housing a regional trauma center and a 154-bed Children's Hospital. The health system averages approximately 21,000 inpatient discharges and 90,000 emergency room visits per year. Medicare and Medicaid together account for over $50 \%$ of the patient care revenues.

In 2016, AU Health faced challenges (similar to those described earlier), with the use and implementation of its EHR MedRec system (which is powered by Cerner Inc., a federally certified EHR vendor). Although MedRec was often marked as "complete" on the EHR, before patient discharge from the hospital, AU Health leadership 
estimated the patient's active medication list to be inaccurate (with discrepancies between patient's home and hospital medication lists, in regard to drugs, dosages, and frequencies), for a majority of discharged cases.

Importantly, there was consensus among senior administrators at AU Health, that the EHR MedRec system was not being used effectively by providers, to communicate changes in the active medication list across the care continuum and to patients/families. This challenge was ascribed to the general reluctance of physicians to discontinue medications that they did not originally order from the active medication list, which led to not only medication discrepancies during transitions of care, but also to frustrations associated with inaccurate and incomplete medication lists, among patients and providers alike. Therefore, AU Health faced a scenario that was reflective of national concerns related to use and implementation of EHR MedRec in hospitals and health systems, ie, low physician engagement, translating to limited use of EHR MedRec technology.

\section{Study purpose}

In fall 2016, Augusta University secured a 2-year grant from the Agency for Healthcare Research and Quality (AHRQ), to pilot a Social Knowledge Networking (SKN) system pertaining to EHR MedRec, to enable AU Health to progress from "limited use" of EHR MedRec Technology, to "meaningful use." "16,17 As discussed earlier, a primary reason identified in the literature for limited use of EHR MedRec technology, is low physician engagement, emanating from an absence of professional consensus (or collective understanding) among providers of 1) the value of EHR MedRec practices in promoting patient safety and 2) the system-level EHR MedRec workflow, including the responsibilities of each provider group in the MedRec process, across the continuum of care.

The rationale for an SKN system is that it could provide a platform for tacit (practice-based) knowledge exchange on issues related to EHR MedRec, across diverse provider subgroups and care settings, to highlight adverse consequences of gaps in practice for patient safety (eg, not using the electronic medication history function resulted in an error in recording dosage upon admission, which resulted in an adverse event for the patient). This, in turn, is expected to increase physician engagement in addressing issues related to EHR MedRec; and promote collective learning of best practices (eg, using the electronic medication history during each encounter, to generate the current medication list), to provide a foundation for practice change (improvement), ie, Meaningful Use (MU) of EHR MedRec technology. This paper discusses the methods and results associated with two specific aims of this study:

1. Examine the dynamics of interprofessional knowledge exchange and learning related to EHR MedRec on the SKN system.

2. Explore associations between "SKN Use" and "Meaningful Use (MU) of the EHR MedRec system," with the latter being assessed in terms of adherence to best practices in EHR MedRec, ie, practices known to reduce medication discrepancies and promote medication list accuracy during transitions of care (as described under "Methodology").

\section{Theoretical framework}

The theoretical foundation for using an SKN system to facilitate provider engagement, interprofessional learning and practice change (ie, MU-of EHR-MedRec), emanates from integrating two literature streams: 1) Professional Complex Systems Theory and 2) Social Network Theory. "Complex systems," refers to organizations composed of many components that interact with one other, to give rise to collective organizational behavior. Complex systems theory has strongly reinforced the message that there can be no organizational change without organizational learning, and organizational learning, in turn, requires the exchange of tacit (practice-based) knowledge, to facilitate engagement and collective learning. ${ }^{18}$ Congruently, the literature on social network theory, has sought to understand which communication network structures may be most effective for tacit knowledge exchange, engagement, learning, and change, in complex systems. Studies in this niche, have put forth that communication networks rich in “density," ie, peer-to-peer networks with limited involvement of administrators may be most effective for tacit knowledge exchange, learning, and change in complex systems. However, this literature has largely been restricted to consulting and business organizations.

Health services researchers have applied social network theory to the professional organizational context, to gain insight into the effective communication network structures in professional complex systems (PCS). PCS are organizations that exhibit the properties of both professional organizations and complex systems, like healthcare organizations (HCOs). This research suggests that networks rich in "brokerage" and "hierarchy," with relatively less "density" across professional subgroups, may be more effective for 
engagement, tacit knowledge exchange, learning, and change in "professional complex systems."19-23 While "hierarchy" refers to hierarchical top-down communication, "brokerage" refers to the ability to transfer knowledge from one subgroup to another, by virtue of having a global view of the discussions.

In other words, this literature suggests that proactive and periodic top-down communications of best practices, may be most effective for enabling engagement and tacit knowledge exchange across professional subgroups, to foster collective learning and enable change in professional complex systems (PCS). The reasoning is that PCS contain multiple professional subgroups with "subgoals" (subgroup goals) that are reinforced through ongoing in-group communication. This results in an absence of cognitive linkages across subgoals, and between subgoals and organizational goals. Therefore, during times of change, senior administrators must undertake proactive and periodic efforts to create cognitive linkages between subgoals and organizational goals, to enable tacit knowledge exchange, engagement, learning, and change in PCS, including HCOs. ${ }^{19-27}$ Therefore, this theoretical framework (summarized in Figure 1) suggests that a Social Knowledge Network (SKN) moderated by senior administrators, to engage provider subgroups in tacit knowledge exchange on practice issues related to EHR MedRec, while also facilitating proactive, periodic, top-down communication of best-practices related to EHR MedRec, can foster collective learning and practice change (eg, Meaningful Use of EHR MedRec technology), in HCOs.

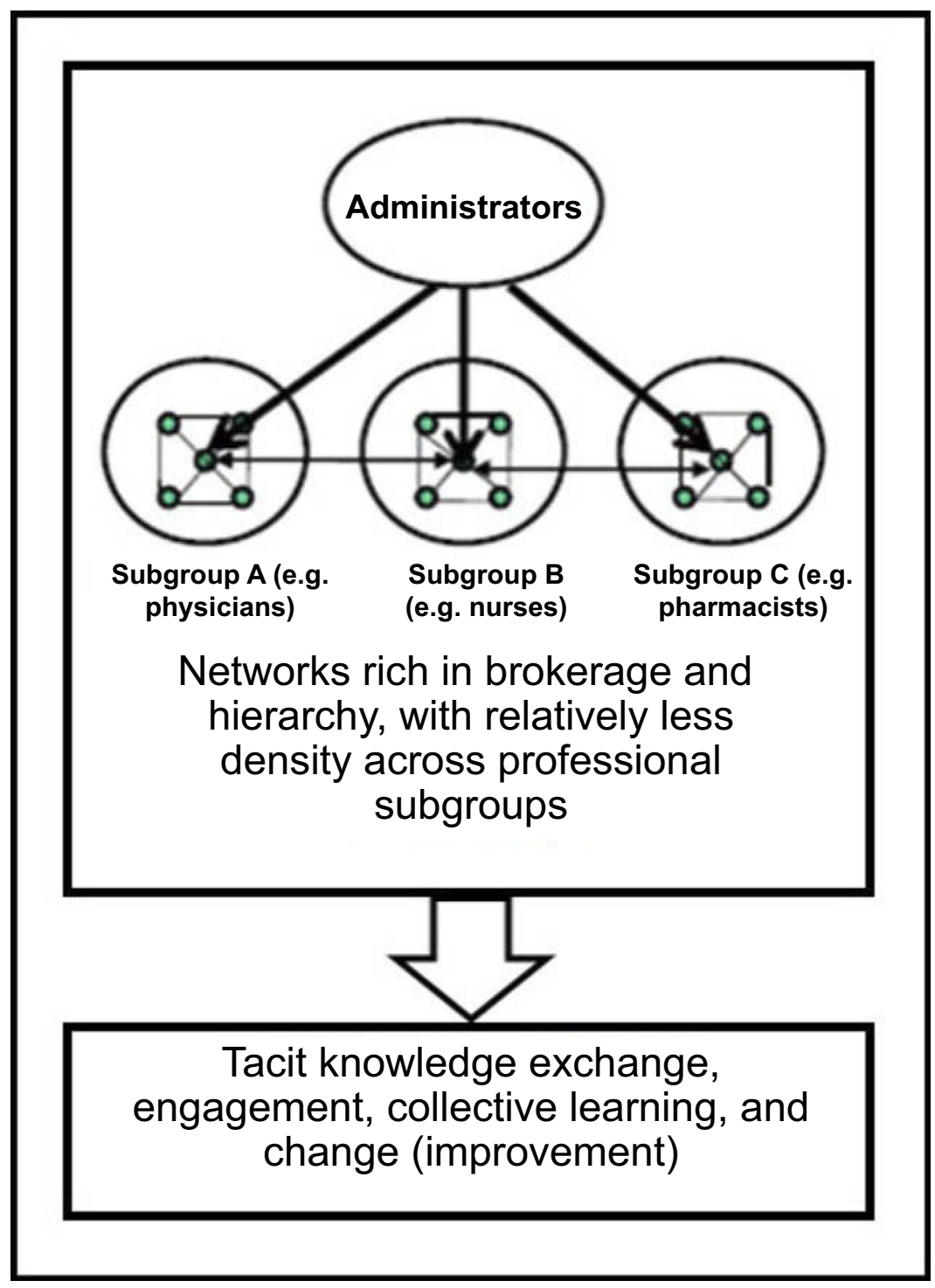

Figure I Effective communication network structure for learning and change in professional complex systems (eg, healthcare organizations). 


\section{Methodology}

An exploratory, qualitative approach was used for data collection and analysis. The crux of the project was a pilot-implementation of a moderated SKN system on issues related to EHR MedRec, over a 1-year period; ie, April 1, 2017 to March 31, 2018 (or Q2 2017 to Q1 2018), among diverse provider subgroups and care settings, ie, physicians, nurses, and pharmacists based in outpatient and inpatient medicine settings at AU Health.

\section{Design and implementation of the SKN system on EHR MedRec}

The SKN system implemented at AU Health included several components:

- SKN Reporting Tool: an online form that allowed participating providers (SKN Users) to report practice issues related to EHR MedRec (eg, challenges in obtaining complete information at admission for compiling the patient's current medication list). The form allowed users to provide a brief description of the issue and indicate the care settings and patient conditions it applies to.

- SKN Discussion Tool (Microsoft Yammer): an online platform, separate from the SKN Reporting Tool, to enable moderated discussions on issues related to EHR MedRec. Microsoft Yammer is an example of an enterprise SKN system. Our study used the basic version of Yammer, which was already available to AU as part of its Office 365 package.

- SKN Lunch-and-Learn Sessions: A total of 5 Lunch-and-Learn sessions were held over the 1-year SKN period, for participants to meet and discuss lessons learned from exchanges on SKN Yammer. Advance invitations to these sessions were sent to all SKN participants.

- SKN Periodic Email Updates: Approximately 15 periodic progress update emails were sent by the Principal Investigator (PI) to all SKN participants, over the 1-year SKN period.

There were two types of participants in the SKN system:

1. SKN Moderators: included a group of 5 senior administrators and provider champions who played a key role in moderating discussions on issues related to EHR MedRec among participants (SKN
Users), over the 1-year SKN period. The 5 SKN moderators included the Chief Medical Officer (CMO), Chief Medical Information Officer (CMIO), two hospitalist chiefs, and the Principal Investigator (PI). A key responsibility of SKN Moderators was to bring issues related to EHR MedRec reported by individual SKN Users on the SKN Reporting Tool, for discussion by all SKN Users on Yammer. As such, a majority of threads of discussion on Yammer began with an issuereport (problem statement) brought to Yammer by SKN Moderators. While SKN Moderators would bring the brief issue-descriptions to Yammer, they would not reveal the identity of the person reporting the issue, to enable SKN Users to maintain a level of confidentiality in reporting issues. Another responsibility of SKN Moderators was to proactively initiate discussions on topics relevant to EHR MedRec (eg, best practices identified in the literature), and bring lessons learned from the SKN system, for discussion at regular health system meetings, including the Hospital Quality \& Patient Safety Council, chaired by the CMO.

2. SKN Users: included 50 practitioners, ie, physicians, nurses, and pharmacists based in outpatient and inpatient medicine services at AU Health, who agreed to participate in the SKN system. Key responsibilities of SKN Users were to 1) report issues related to EHR MedRec on the SKN Reporting Tool on an ongoing basis; 2) participate in moderated discussions on SKN Yammer, over the 1-year period; and 3) share lessons learned from their ongoing participation on the SKN, with colleagues in the health system. However, SKN Users were not allowed to initiate new threads of discussion directly on SKN Yammer. This ability was restricted to SKN Moderators, to enable coordinated discussions of issues related to EHR MedRec on the SKN system.

\section{Participant recruitment and orientation}

Participant recruitment to the SKN system, began after the project received IRB approval from Augusta University. All practitioners in three professional subgroups, ie, physicians, nurses, and pharmacists, within five inpatient and outpatient medicine services at AU Health, including Cardiology, Internal Medicine, Family Medicine, Emergency Medicine, and Hospitalist service lines, were approached 
by the PI for recruitment using the IRB-approved informed consent process. A total of 50 practitioners were recruited to participate as SKN Users, including 15 physicians, 15 nurses, and 20 pharmacists, from outpatient and inpatient medicine settings. All participating physicians from Cardiology, Internal Medicine, and Family Medicine practiced in both inpatient and outpatient settings. Only Hospitalists were exclusively inpatient practitioners. It would be relevant to note that there were a total of $\sim 200$ eligible practitioners (physicians, nurses, and pharmacists) within the five targeted medicine service lines at $\mathrm{AU}$ Health. As such, by recruiting 50, we achieved a dual goal of 1) reaching our recruitment target for this pilot study and 2) attracting a critical mass of over one-fourth of the total number of eligible practitioners at AU Health.

Everyone who agreed to participate, was requested to review and sign: 1) an Informed Consent document; 2) a Statement of Professional \& Ethical Conduct on SKN; 3) an SKN Privacy \& Confidentiality Agreement, which stressed that use of Protected Health Information (PHI) anywhere on the SKN system, was strictly prohibited; and 4) a Custom Usage Policy, which clarified that SKN Users were not allowed to initiate threads of discussion directly on SKN Yammer; they would only report issues through the SKN Reporting Tool. For coordination purposes, only SKN Moderators would have the ability to initiate threads of discussion on Yammer. Participants then received a detailed online orientation to the SKN system, prior to its launch, including steps for accessing the SKN Reporting Tool and SKN Yammer, both of which, were made available to participants through separate links within the EHR. Both tools were also accessible through the enterprise employee web portal and mobile devices; Yammer was downloadable as an App. A participant engagement plan, including follow-up emails to participants and quarterly distribution of $\$ 25$ gift cards to selected participants, was implemented over the 1-year period, to ensure that the SKN, was being used for its intended purpose.

\section{Data collection and analysis Data collection}

SKN Use: Data on SKN Use were collected over the 1year SKN period. A key data source was Yammer, which included a record of all communications posted by SKN Users, on various threads of discussion related to EHR MedRec. A majority of these threads stemmed from issues reported by SKN Users via the SKN Reporting Tool. All threads of discussion on Yammer, were open and available to all SKN Users, without restriction. Yammer recorded the name, date, and time associated with each posting. Additional data sources related to SKN Use included the SKN Reporting Tool; the 15 SKN progress email updates; and the 5 SKN Lunch-and-Learn sessions, which were held in April 2017 (Quarter 1 or Q1 of SKN period), July 2017 (Q2), October 2017 (Q3), February 2018 (Q4), and May 2018 (after SKN implementation). Each SKN Lunch-and-Learn session lasted $\sim 60$ mins and was attended by $\sim 20$ participants. All sessions were audiorecorded and transcribed to text, to enable Thematic Analysis.

Meaningful Use (MU) of EHR MedRec: MU of EHR MedRec was measured in terms of adherence to two best practices in EHR MedRec, ie, practices known to reduce medication discrepancies and promote medication list accuracy during transitions of care. MEASURE 1 and MEASURE 2 are outlined below. It would be relevant to note that due to the exploratory nature of the study, the decision to use these measures emerged from the interprofessional knowledge exchange dynamics on the SKN system, during the first few months of the SKN period. Since both were practice measures, they could be captured retrospectively from the EHR. Data on these measures were collected over 6 quarters, ie, Q1 2017 through Q2 2018. This translated to one quarter before SKN was launched, extending to one quarter after SKN was concluded:

MEASURE 1: External Rx History Import (Higher is Better): Defined as the aggregate proportion of patient encounters during which the External Rx History was imported at some point before the encounter ended (inpatient or outpatient). Data were obtained at the encounter level, quarterly, for 6 quarters, for each of the 5 medicine services represented on the SKN, and aggregated, to examine trends in the proportion of External Rx History Import. Since Cardiology was the only medicine subspecialty represented on the $\mathrm{SKN}$, this measure was restricted to patient encounters for a Cardiology-relevant chronic condition, ie, patients who had a primary or secondary diagnosis of Congestive Heart Failure (CHF), based on ICD-10 codes.

"External Rx History Import" refers to the importation of the patient's medication history filled at their pharmacy. The External Rx History can be imported when the provider (physician, nurse, or pharmacist) activates the External Rx History button on the EHR, which would access the Surescripts system to pull the patient's Rx history. Surescripts is an IT company that supports e-prescription, 
the electronic transmission of prescriptions between HCOs and pharmacies. According to the US DHHS, in 2014, $96 \%$ of the US community pharmacies used the Surescripts network. ${ }^{28}$ At AU Health, it was determined that $90 \%$ of the patients fill their prescriptions at pharmacies that participate in the Surescripts system. "External Rx History Import" (MEASURE 1) is a measure of MU of EHR MedRec technology, because a higher proportion reflects better use of the EHR system by providers, to obtain a current medication list, for reconciling with new prescriptions, to reduce medication discrepancies and increase accuracy of the medication list, during transitions of care.

MEASURE 2: Missing Documentation of Compliance Status (Lower is Better): Defined as the aggregate proportion of patients' total active medications that are missing documentation of compliance status. Data for this measure were collected at the patient level, quarterly, for 6 quarters, for all patients who had a primary or secondary diagnosis of $\mathrm{CHF}$, and at least one encounter with any one of the 5 medicine service lines represented on the SKN, over the 6-quarter period. There were a total of 2,846 individual patients who met these criteria.

The aggregate proportion of missing compliance status documentation was calculated for all patients with reported active medications by quarter, to examine trends over the 6-quarter period. It is essential for all medications on a patient's active list to have a documented (non-missing or non-blank) compliance status so that the next provider is equipped with information needed to accurately update the list, before the subsequent transition point. For example, if compliance status for a medication is "unable to determine" in the emergency department, the admitting hospitalist might talk to the patient/family to update the status to "still taking as prescribed." On the other hand, if the medication is determined to be "not taking," because its course has been completed, then it could be removed from the list, prior to discharge. "Missing Compliance Status Documentation" (MEASURE 2) is a measure of MU of EHR MedRec technology, because a lower proportion reflects better use of the EHR system by providers, to communicate changes in the patient's active medication list, across the continuum of care, in an effort to reduce medication discrepancies and promote accuracy of the medication list during transitions of care.

In summary, both MEASURE 1 and MEASURE 2 reflect effective use of EHR MedRec system functionalities by providers, to reduce medication discrepancies and improve medication list accuracy. It would be relevant to note that both measures are designed to capture practices implemented by a team of providers, ie, they could be completed by a physician, nurse, or pharmacist in a particular service line, caring for the patient in the hospital or clinic. As such, it would not be feasible to track adherence to these practices for patients cared for only by the specific providers who participated on the SKN system. On the other hand, it would be both feasible and valid to track adherence to these practices for groups of patients with chronic conditions (like CHF), cared for by the medicine service lines represented on SKN, for three reasons: 1) SKN participants by themselves are expected to touch many patients within these services; 2) SKN participants constituted a critical mass of the total eligible medicine provider group; and 3) knowledge exchanged on SKN was expected and encouraged to be spread to peers, especially within participants' own service lines, since SKN exchanges were directly relevant to daily care practices in the hospital and clinics.

\section{Data analysis}

To address Aim \#1, we examined dynamics of interprofessional knowledge exchange on the SKN system, using data from Yammer, supplemented with data from SKN Lunchand-Learn sessions and SKN progress email updates. We began by conducting Thematic Analysis of data from Yammer, using QSR NVivo $14 .^{29}$ The raw Yammer dataset consisted of a total of 485 posted communications, divided into 62 threads of discussion (distinguished by Thread ID). The entire dataset was organized by thread, in chronological order, which enabled Thematic Analysis to be conducted sequentially by thread, to understand dynamics of knowledge exchange, both by thread-of-discussion, and broadly, for the entire dataset.

The analysis team consisted of four researchers, 1 medical doctor (MD), 1 health service researcher $(\mathrm{PhD})$, and 2 Graduate Research Assistants in the applied health sciences. To begin with, the first two researchers completed all key phases of Thematic Analysis, to develop an initial coding scheme for identifying key themes. ${ }^{30,31}$ This included: 1) data familiarization; 2) search for themes; 3) review of themes; 4) defining and naming themes; and 5) generation of the initial coding scheme, including the "theme-node hierarchy" on NVivo. The initial coding scheme was used by the other two researchers to code approximately $33 \%$ of the raw dataset from SKN Yammer. Analysis of initial intercoder agreement 
amongst the four researchers revealed a 70\% match. Follow-up discussions amongst the four researchers resulted in further refinements to the coding scheme.

The revised coding scheme was used by all four researchers to recode entire raw dataset. This iterative process of coding, evaluation, discussions, and refinements to the coding scheme was repeated until intercoder agreement of over $95 \%$ was reached among four researchers. There were six broad themes (or categories) of interprofessional knowledge exchange identified from this process that are elaborated further under Results: "Problem Statements;" "Problem-Solving Statements;" "IT System Education;" "Best-Practice Assertions;" "Culture Change Assertions;" and "Collective Learning (Aha) Moments." The next step in the process, was Thematic Analysis of content transcribed from the 5 SKN Lunch-and-Learn sessions and 15 SKN progress email updates, by the same coding team, using the final coding scheme developed for Yammer. This process revealed that no further changes were needed to the coding scheme.

To address Aim \#2, we extracted and analyzed longitudinal patient-level data on MEASURE 1 and MEASURE 2 from the AU Health EHR, with support from a health system analyst. We performed trend analysis of quarterly data on both measures, to understand patterns over time. We examined associations between SKN Use and MU of EHR MedRec, by placing timestamps on trend charts to represent culminating points in interprofessional learning associated with the two measures, identified from Thematic Analysis, eg, "Collective Learning (Aha) Moments" experienced during the SKN Lunch-and-Learn sessions. For MEASURE 1 (External Rx Import), we also analyzed comparative data for all patient encounters in the Neurosurgery service line, which was not represented on the SKN system. Comparative data were not available for MEASURE 2 (Missing Compliance Status Documentation).

\section{Results}

\section{Dynamics of interprofessional knowledge exchange and learning on the SKN system (Aim I)}

Of the 50 practitioners who signed up to SKN Users, 25 were active users of SKN Yammer, with 12 or more posts over the 1-year SKN period. Active users of Yammer came from all professional subgroups and care settings represented on SKN, including 8 physicians, 9 nurses, and 8 pharmacists. Additionally, 3 of the 5 SKN Moderators (including the $\mathrm{CMO}, \mathrm{CMIO}$, and $\mathrm{PI}$ ), each posted 40 or more messages on Yammer, over the 1-year SKN period.

As indicated earlier, over the 1-year SKN period there were a total of 485 posted communications on Yammer, divided into 62 threads-of-discussion. Of these, 45 threads had 3 or more posts; among which, 12 threads had 10 or more posts; of which 3 threads had 25 or more posts, including 1 thread with 45 posts. There were a total of 32 issues related to EHR MedRec reported on the SKN Reporting Tool, over the 1 -year period. Of the 45 threads with 3 or more posts, 32 began with issues reported on the SKN Reporting Tool, brought in to Yammer by SKN Moderators. As such, all reported issues were used to launch threads of discussion on Yammer.

Overall, the Thematic Analysis process identified six broad themes that were repeated across several threads of discussion, in the chronological order (outlined below):

1. Problem Statements

2. Problem-Solving Statements ("The How-To")

3. IT System Education ("The What")

4. Best-Practice Assertions ("The Why")

5. Culture Change Assertions ("The Way-To")

6. Collective Learning (“Aha”) Moments

When the entire Yammer dataset and supplemental sources on SKN use (ie, Lunch-and-Learn sessions and progress email updates) were considered, there were several layers of sub-themes under each of the six broad themes. Figure 2 summarizes this hierarchy of themes. In essence, this figure provides a foundation for broadly articulating the dynamics of interprofessional knowledge exchange related to EHR MedRec, on the SKN system.

A majority of issues related to EHR MedRec that were used to launch threads of discussion on Yammer (from the SKN Reporting Tool), pertained to the broad challenge of "communication across the provider continuum," within the context of "medication-list accuracy." To elaborate, one of the most voluminous threads (THREAD 1) began with a problem statement on obtaining medication history from the patient upon arrival, to formulate the current medication list, for reconciliation with new prescriptions, to create an accurate updated active medication list on the EHR. Another voluminous thread (THREAD 2) began with a problem statement on how medications that do not belong in the active medication list, do not get removed at discharge, thereby reducing the accuracy of the patient's active medication list on the EHR. As such, 


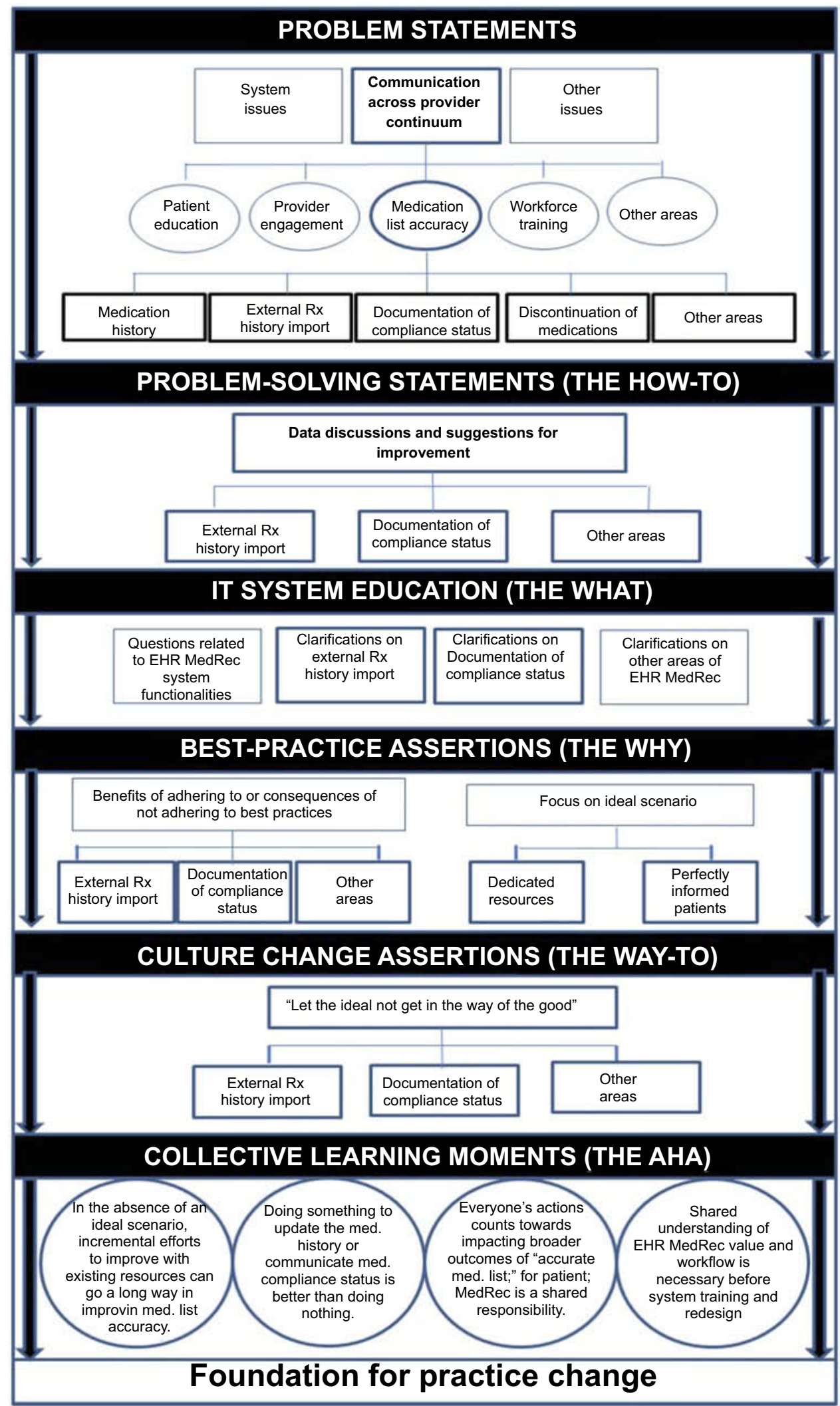

Figure 2 Dynamics of interprofessional knowledge exchange related to EHR MedRec on the SKN system. 
two of the most voluminous threads on Yammer, pertained to problem areas most frequently encountered by practitioners in context of EHR MedRec, at a national level, as reported in the literature. ${ }^{1,2}$

\section{Dynamics of interprofessional knowledge exchange and learning in THREAD I}

THREAD 1 began with the problem statement from an Emergency Department (ED) nurse "Patients frequently arrive to the ED with no medication list and they don't know the names of all or some of their medications .... The nurses typically attempt to put in what they know or have but it is incomplete a lot of the times...." This concern was echoed by others. For example, a Cardiologist shared the following from an outpatient clinic perspective: "Yesterday, I had a new patient who was on many medications but had neither the bottles nor a list. I cannot bill until meds are reconciled and yet this cannot be honestly done...."

This was followed by problem-solving statements; spinoff problem statements; and additional problem-solving statements. For example, an outpatient nurse then put forth a problem-solving statement suggesting use of the External Rx History Import option, to obtain a current list of medications. "We use the External Rx History option to populate the medication list when we have a NEW patient that does not bring their pill bottles. We have found this option to be helpful. Does ED have access to this system? It would at least give an idea of meds patient is taking and it does show last time med was refilled." This suggestion initially received dissension from participants who had originally reported the problem. For example, the ED nurse shared: "Yes we have the external history option, but it isn't as easy as one might think. You have to take the time to compare what is in the system to what is in the external history, and when they don't match up, you have to ask the patient .... some are insinuating that they will "trust" the history in the system, but it could be inaccurate." The Cardiologist chimed in to say: "Patients should bring in all home medications. I think importing the data in the absence of seeing the pill bottles creates errors." This was then followed by additional problem-solving statements. For example, a Hospitalist commented: "I think there are few of us who fully trust any electronic list of meds. Usually I use it as a template to start discussing with patients what they're actually taking. Then once you have accurate medication lists the real question is compliance."

These discussions were followed by "system education" from the CMIO/SKN Moderator. "The External
Med History is an option on the Medication List page. This option shows last refill and often the prescriber. MDs can use this function to convert medication to prescription as well to populate medication list."

These discussions progressed to best-practice assertions, which encountered some initial dissension, with focus on the ideal scenario. For example, a pharmacist put forth a best practice assertion: "Importing 'External Rx History' at some point in the patient encounter, preferably as close to admission as possible, is essential as it includes $90 \%$ plus of what actually occurred in the community. We all know there will be gaps (i.e. VA, cash paying, patient assistance medications, etc.). However, it will at least allow providers to know what the patient has been prescribed and could potentially question why they are not taking certain medications." Similarly, the CMO/ SKN Moderator shared: "I am reviewing a case. Had the provider checked the external history, a verbal miscommunication would not have resulted in a dosing error." Despite these best-practice assertions, there was continuing disagreement with a focus on the ideal scenario: "I think the only way to accurately reconcile medications is to see all the bottles the patient has." There were also comments on the need for dedicated resources: "I think the only way the ED can make this work would be to have help from pharmacy techs dedicated to only this task." It would be relevant to note at this juncture, that AU Health has dedicated pharmacists and/or pharmacy techs in several outpatient and inpatient areas, but not in all departments.

A pharmacist then put forth a culture change assertion. "It is important not to let the perfect stand in the way of the good or better, I have seen prescribers simply 'not act' to reconcile a medication because there was insufficient information or they were not sure. Not acting is making a decision. (to do nothing)." The CMO/SKN Moderator then reinforced this message with some comments: "Many ED patients and even hospital-to-hospital transfers have little ability to give a med. history. That is why External $\mathrm{Rx}$ is so important. While not perfect, it does give fills of prescriptions with fill dates, and we have seen events that would have been prevented if this was done." Regarding the issue of adding Pharmacy Techs to ED, the CMO/SKN Moderator commented: "we would not be good stewards of our resources, unless we optimize the workflow and standardize it. Several of our clinics have already implemented these best practices and we need to spread them. Further role clarification is the key. Only after we do that 
would we know the resources needs. For example, a key process for our techs is to call pharmacies to obtain information. The best source even for them is the External $\mathrm{Rx}$ History since there is a lag in obtaining information from many pharmacies that do not operate $24 \times 7$."

The PI/SKN Moderator then posted the following collective learning (aha) moment in an effort to synthesize lessons learned: "Our discussions suggest that incremental efforts to improve medication history with External $\mathrm{Rx}$ History Import, in the absence of dedicated resources, can go a long way in increasing accuracy of the medication list."

The second SKN Lunch-and-Learn session held at the start of Q2 of the SKN period was devoted to discussing lessons learned on Yammer related to "External $\mathrm{Rx}$ History Import" on the EHR, a topic that laid at the core of the Yammer discussions in THREAD 1 (as described above). Correspondingly, this Lunch-and-Learn session was significant in providing an opportunity for additional collective learning on this topic. Several participating nurses and pharmacists who had used the External Rx History function articulated how helpful it was in obtaining an initial medication history for supplementing with information from the patient/family, to develop a current medication list for reconciliation.

This discussion culminated in additional collective learning (aha) moments, summarized by the PI at the end of the session: "Lessons learned from Yammer, suggest that in the absence of an ideal scenario (e.g., dedicated resources for EHR MedRec or perfectly informed patients with all pill bottles), incremental efforts to improve practices with existing resources (e.g., External Rx Import), could go a long way in reducing medication discrepancies during transitions of care. In other words, doing something to reconcile medications (using External Rx Import), is better than doing nothing, for improving accuracy of the medication list across the care continuum, to promote patient safety."

\section{Dynamics of interprofessional knowledge exchange and learning in THREAD 2}

THREAD 2 began with a problem statement from an outpatient nurse: "We are coming across multiple patients that were on two beta blockers. They had been switched from metoprolol to carvedilol or vice versa but both meds remained on their med list and the issue was not known until patients would call and state they were feeling very fatigued and heart rate was low - then we start investigating." Another pharmacist echoed this concern: "As new prescriptions are being written, the old ones are not being discontinued on the history. To tie into this issue, I have had several patients contact me asking to remove a medication from their list that they have never been prescribed."

This was followed by problem-solving statements; spin-off problem statements; and additional problem-solving statements. An outpatient nurse then advanced an initial problem-solving statement for using the "Compliance Status" field, to indicate "Not Taking" with additional notes as needed, to indicate formulary replacement or completed course; and just removing the medication. "If the patient is no longer taking the medication, why not just right click and select complete and it's gone? You could notate the reason for removing prior to completing under Compliance Status so that when the next person reviews inactive/discontinued meds they'll see the rationale." However, a Hospitalist physician had a disagreement: "There are cases that patients are no longer taking medications but probably need to be taking them. In such cases, discontinuing the medication would not be appropriate." This was followed by additional problemsolving statements from a pharmacist: It would be helpful to have 'not taking - told to stop by doctor' versus 'not taking - non-compliance' in addition to 'not taking unable to determine' which we already have, to provide more information into what barriers/issues exist for adherence and access."

The CMIO/SKN Moderator then stepped in with some system education: "Under Compliance Status, 'Not Taking' was meant for meds that the patient was supposed to be taking but are not compliant with. Intake staff were supposed to "complete" meds that had been discontinued by a provider or that the patient self-discontinued. Of course there is some gray area between the two, which would be a clinical judgement call."

These discussions progressed to best-practice assertions, which encountered some initial dissension, with focus on an ideal scenario. For example, there were several best-practice assertions from pharmacists and nurses, to reinforce the benefits of documenting compliance status for every active medication; as well as the consequences of not doing so: "A correct medication list is essential for a patient to move from hospital to the community physician. Discontinuing 'Not Taking' Medications needs to occur at the point of entry or exit from the acute care stay. As such, completing the Compliance Status for every medication during every encounter is essential. Compliance status can be assessed 
by fill history and asking the patient how they take their medications. (e.g., if prescription is for Lisinopril $20 \mathrm{mg}$ daily, \#30, for 12 refills; and fill history only shows one fill over 6 months, then patient is either non-compliant or was told to stop taking the medication)." Another pharmacist commented: "The issue generally starts with the medication plan being verbally communicated to the patient and not completely written down. In such cases, the lists would not include 'the following medicines have been discontinued,' which in turn, leads to therapeutic duplication." Concurrently however, there were comments from other pharmacists that stressed the need for dedicated resources "I have read that pharmacy techs are very effective in solving these problems as they are familiar with the medications, regimens, refill patterns, etc. This effectiveness comes from looking at the information on a daily basis."

Next, a pharmacist who made a similar comment in the context of the discussion on External Rx History Import put forth a culture change assertion. "Again, it is important to not let the perfect be the enemy of the good. I agree that if the Compliance Status is 'not taking' anymore, that needs to be flagged so the next prescriber is aware and can consider the information (eg not ordering a medication for a patient who has not taken for years - AND removing the med from the list of active medications). This is NOT prescribing, but should be done with a note on the specifics - eg "stopped due to side effects" versus "the specialist who prescribed it told me to stop." These have different implications that the clinician needs to consider)." Having partial information on the drug name is better than no information at all, as long as this is flagged by the team for follow-up, eg, for an inpatient admission that might be asking the family to bring the $\mathrm{Rx}$ in, or calling a physician's office/pharmacy the next morning." These comments were then reinforced by the CMO/SKN Moderator: "To require all areas documented would put the documenting individual in a tough position. Sometimes you do not know at the time what dose is! What do you do? Do not document the med at all? Documenting the med at least allows interaction checks. So as $<$ the pharmacist> said, let's not allow perfection to become the enemy of good. The key is to teach the importance of compliance and how to document it."

The PI/SKN Moderator then posted the following collective learning (aha) moment comment, in an effort to synthesize lessons learned from the discussion: "This discussion illustrates how the actions of each provider or clinic in completing or not completing the Compliance
Status field, can impact the accuracy of the patient's medication list and ultimately, the safety of the patient as s/he transitions into the community."

The third and fourth SKN Lunch-and-Learn sessions held during the 3rd and 4th quarters of the SKN period, respectively, were devoted to discussing lessons learned on Yammer related to "Documentation of Compliance Status," a key EHR MedRec functionality that laid at the core of Yammer discussions in THREAD 2 (as described above). It would be relevant to note at this juncture, that around end of Q2 of the SKN period (ie, 6 months into the SKN period), data collected up to that point, on the two measures of MU of EHR MedRec, began to be shared by SKN Moderators, with SKN Users, on Yammer. By that point, data on MEASURE 1 had already begun showing improvements, much to the appreciation of Yammer participants. By contrast, however, data on MEASURE 2 showed considerable room for improvement with a growing proportion of missing compliance status documentation, on patients' active medications. Supplemental chart audits that were performed by the CMO and CMIO/SKN Moderators around the same timeframe, revealed a tendency to document only the "not taking" medications by exception in the compliance status field, while leaving other active medications, with missing or blank compliance status. These findings were shared with all SKN Users on Yammer. As indicated in Figure 2, any postings on Yammer related to data on the two measures were coded under the sub-theme of "Data Discussions and Suggestions for Improvement," under the broader theme of "Problem-Solving Statements."

During the third SKN Lunch-and-Learn session, a key topic for discussion was the importance of not leaving the compliance status missing or blank for any active medication, since a blank field could be construed to mean either that the patient is "still taking the medication as prescribed;" or that the previous provider was "unable to determine" the status, making it all the more difficult for the next provider of care to trust the data in the EHR, and use it meaningfully to update the medication list for the subsequent transition point. It was discussed that these types of issues could have the effect of prompting workarounds by providers, to communicate any changes to the medication list directly to the patient, either verbally, or on paper, without updating the electronic list on the EHR. These points were met with agreement from providers across the continuum. For example, an outpatient nurse coordinator commented: "I have instructed my staff to address each medication with a 
compliance.” A Hospitalist physician commented: "I agree. I would also like to see system-wide standardization of this process and education of responsible staff."

The next (fourth) SKN Lunch-and-Learn session, culminated in several "Collective Learning (Aha) Moments" on this topic, summarized by the PI at the end of the session: "Our discussions on Yammer suggest that providers across the continuum need to recognize the importance of shared ownership of the EHR MedRec system, since every provider's actions (e.g., compliance status documentation), could affect the accuracy of the patient's active medication list on the system. Our experience with Yammer over the past several months also suggests that developing a collective understanding of both the value of best-practices in EHR MedRec and the EHR MedRec workflow at a system level, is essential, before embarking on IT-training for providers to address socio-technical challenges of EHR implementation (like managing differing 'views' of EHR MedRec functionalities). This shared understanding of the 'big picture' is essential to have in place, before IT-training of providers, to ensure effective communication related to the active medication list across the provider continuum, and promote Meaningful Use of EHR Med Rec technology."

\section{Associations between "SKN Use" and "Meaningful Use (MU) of EHR MedRec" (Aim 2)}

Results for MEASURE 1 and MEASURE 2 are summarized in Part A and Part B, respectively (Figure 3). The graph in Part A summarizes the trend in MEASURE 1, ie, total proportion of External Rx History Import for all $\mathrm{CHF}$ encounters aggregated for the 5 participating medicine service lines, by quarter, over a 6-quarter period (Q1 2017-Q2 2018). The table in Part A indicates the numerators and denominators used to generate the proportions represented on the graph. Both the graph and table in Part A, also include comparative data on MEASURE 1, for all encounters in the Neurosurgery service line, over the same timeframe.

As indicated, the aggregate proportion of External $\mathrm{Rx}$ History Import for $\mathrm{CHF}$ encounters in the 5 participating medicine service lines, increased (improved) from $35 \%$ in Q1 2017 to $84 \%$ in Q2 2018, a $140 \%$ increase. The vertical dotted line on the graph represents the time point of the second SKN Lunch-and-Learn session (ie, end of Q2/start of Q3 2017). This session was conducted after several weeks of interprofessional discussion on this topic on SKN Yammer.
The graph in Part A shows substantial improvement in MEASURE 1 from 59\% in Q2 2017 to 85\% in Q3 2017. On the other hand, the comparative data for Neurosurgery shows a relatively stable trend in MEASURE 1, with the aggregate proportion of External Rx History Import remaining at an average of $\sim 45 \%$, during the same timeframe. These dynamics suggest that the improvements in MEASURE 1 among the participating medicine service lines may have emanated from the interprofessional learning associated with this best practice that occurred on the SKN system.

Similar results were observed for MEASURE 2. The graph in Part B, Figure 3 shows the trend in MEASURE 2, by quarter (from Q1 2017 to Q2 2018). The trend in MEASURE 2 is depicted separately for four groups of patients, distinguished by their total number of active medications in each quarter, over the 6-quarter period. In other words, patients who had $<5$ (Group 1); $\geq 5$-to- $<10$ (Group 2); $\geq 10$ (Group 3) total number of active medications in each quarter; and all patients with reported active medications (Group 4) in each quarter, over the 6-quarter period. The table in Part B shows the numerators (number of medications with missing compliance documentation) and denominators (total number of active medications), used to generate the proportions represented on the graph for MEASURE 2, for each group by quarter; as well as the patient count in each group, by quarter. The two vertical lines on the graph indicate the timings of the third and fourth SKN Lunch-and-Learn sessions, respectively.

As indicated in Part B, MEASURE 2 showed an increasing (deteriorating) trend for all four groups from Q1 2017 to Q4 2017, during which time, an increasing trend was also noted in the total number of active medications among CHF patients (see table in Part B). However, MEASURE 2 began declining (improving) for Group 1, after Q4 2017. In other words, it went from 31\% in Q4 2017 to 17\% in Q2 2018 for Group 1; a $45 \%$ decline (improvement). As indicated on the graph, Groups 2, 3, and 4 also began showing a decline after Q1 2018, ie, one quarter after Group 1. However, the decline for Groups 2, 3, and 4, was not as sharp as it was for Group 1. The earlier start of decline (improvement) in MEASURE 2 for Group 1, compared to the remaining groups, suggests that the interprofessional learning that occurred related to this best practice during the third SKN Lunch-and-Learn session, may have translated to immediate implementation of this bestpractice among patients for whom it could be easily implemented, ie, patients with $<5$ total active medications (the "lowhanging fruits"). However, the fact that there was a declining trend for all groups in Q2 2018 (relative to the increasing trend 


\section{PART A}

\section{MEASURE 1: External Rx History Import (Higher is Better)}

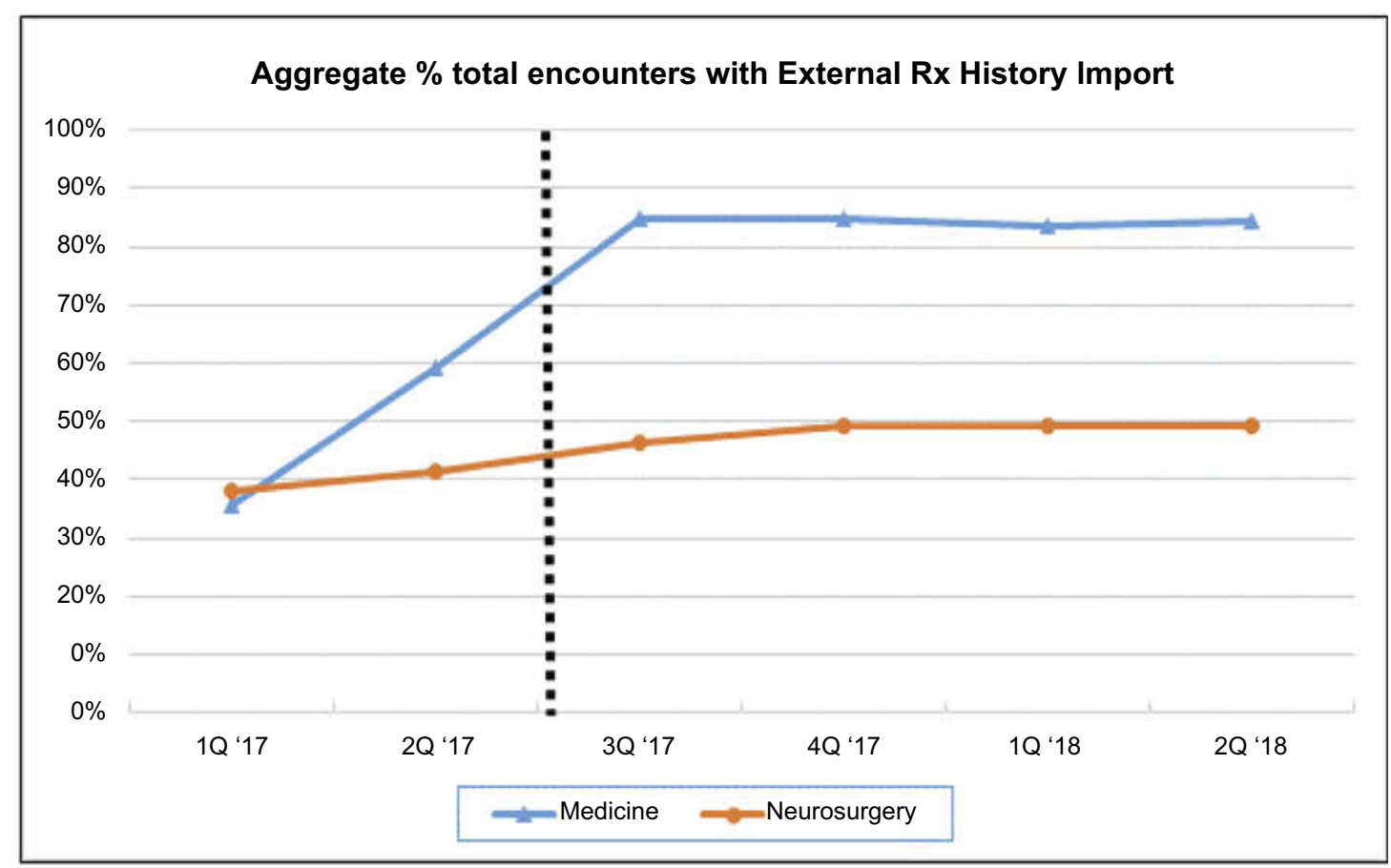

NOTE: The vertical dotted line on the graph, represents timing of the $2^{\text {nd }}$ SKN Lunch-and-Learn session

\begin{tabular}{|l|c|c|c|c|c|c|}
\hline & $1 \mathrm{Q} \cdot 17$ & $2 \mathrm{Q} \cdot 17$ & $3 \mathrm{Q} \cdot 17$ & $4 \mathrm{Q} \cdot 17$ & $1 \mathrm{Q} \cdot 18$ & $2 \mathrm{Q} \cdot 18$ \\
\hline $\begin{array}{l}\text { Number of external Rx history imports } \\
\text { (All five medicine sercvice lines) }\end{array}$ & 455 & 706 & 1,063 & 1,027 & 1,123 & 1,215 \\
\hline $\begin{array}{l}\text { Total number of congestive heart } \\
\text { failure (CHF) encounters } \\
\text { (All five medicine service lines }\end{array}$ & 1,286 & 1,193 & 1,254 & 1,212 & 1,343 & 1,441 \\
\hline $\begin{array}{l}\text { Aggregate proportion of external Rx } \\
\text { history import (Medicine) }\end{array}$ & $35 \%$ & $59 \%$ & $85 \%$ & $85 \%$ & $84 \%$ & $84 \%$ \\
\hline $\begin{array}{l}\text { Number of external Rx history imports } \\
\text { (Neurosurgery service line) }\end{array}$ & 452 & 441 & 456 & 494 & 547 & 496 \\
\hline $\begin{array}{l}\text { Total number of encounters } \\
\text { (Neurosurgery service line) }\end{array}$ & 1,186 & 1,064 & 988 & 1,000 & 1,113 & 1,005 \\
\hline $\begin{array}{l}\text { Aggregate proportion of external Rx } \\
\text { history import (Neurosurgery) }\end{array}$ & $38 \%$ & $41 \%$ & $46 \%$ & $49 \%$ & $49 \%$ & $49 \%$ \\
\hline
\end{tabular}

Figure 3 Trends in measures of Meaningful Use (MU) of EHR MedRec technology.

in previous quarters), suggests that the trend in MEASURE 2 may be moving in the right direction for all patients, although there may be a longer learning curve for patients with more number of total active medications. As such, similar to MEASURE 1, the improvement in MEASURE 2 for the medicine services represented on SKN, coincided with the timeframe during which interprofessional learning on this topic, occurred on the SKN system.

\section{Discussion}

Over the 1-year SKN period, there were two areas related to EHR MedRec (1) External Rx History Import and (2) 


\section{PART B}

\section{MEASURE 2: Missing Compliance Status Documentation (Lower is Better)}

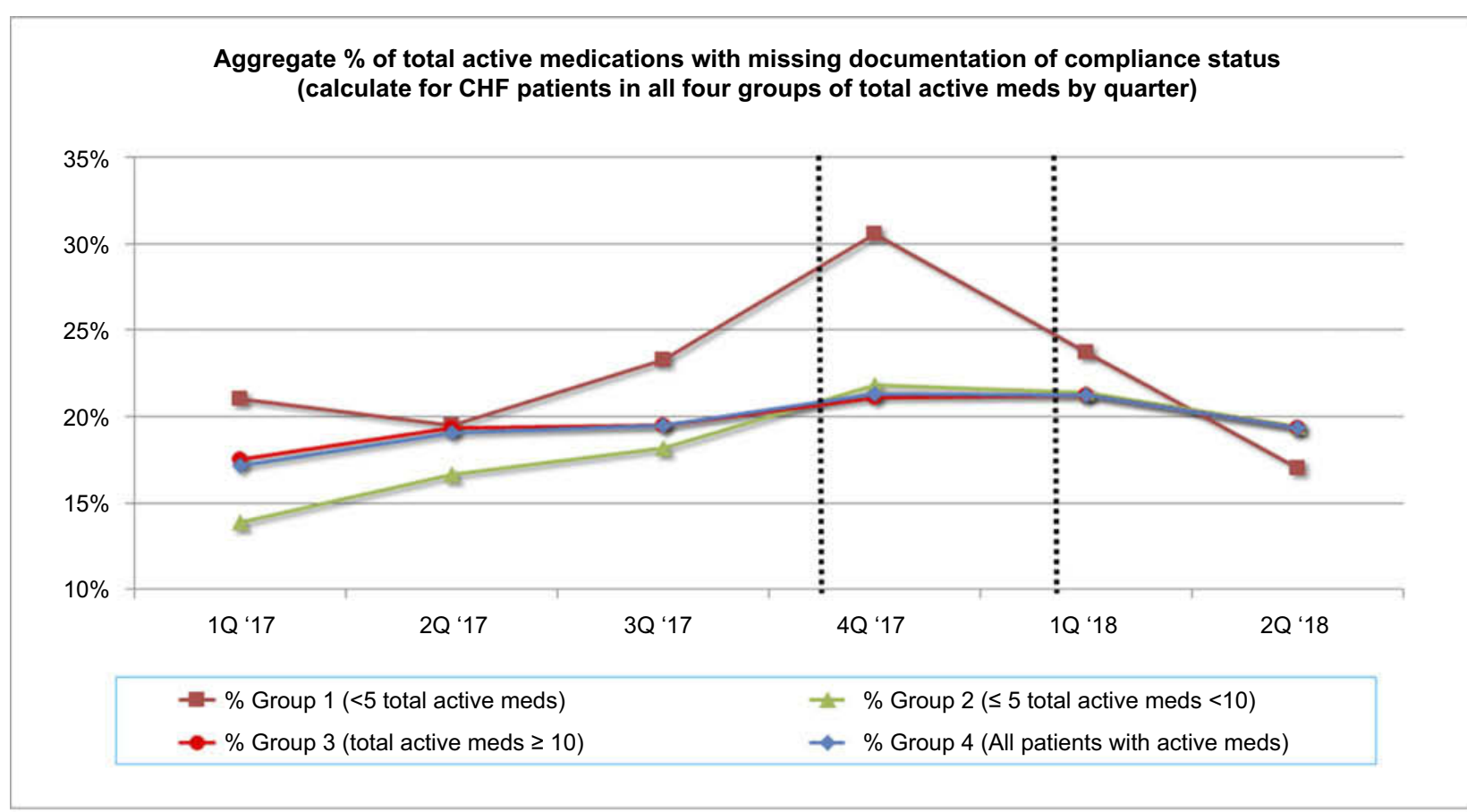

NOTE: The two vertical dotted lines on the graph represent timings of the $3^{\text {rd }} \& 4^{\text {th }} S K N$ Lunch-and-Learn sessions

\begin{tabular}{|l|c|c|c|c|c|c|}
\hline & $1 \mathrm{Q} \cdot 17$ & $2 \mathrm{Q} ' 17$ & $3 \mathrm{Q} \cdot 17$ & $4 \mathrm{Q} \cdot 17$ & $1 \mathrm{Q} \cdot 18$ & $2 \mathrm{Q}$ '18 \\
\hline Number of meds missing compliance documentation in group 1 & 160 & 150 & 155 & 182 & 111 & 55 \\
\hline Number of meds missing compliance documentation in group 2 & 160 & 448 & 505 & 590 & 461 & 263 \\
\hline Number of meds missing compliance documentation in group 3 & 2,591 & 3,900 & 5,240 & 7,262 & 9,251 & 11,071 \\
\hline Number of meds missing compliance documentation in group 4 & 3,086 & 4,498 & 5,900 & 8,034 & 9,823 & 11,389 \\
\hline Total number of active meds in group 1 & 761 & 771 & 666 & 595 & 463 & 324 \\
\hline Total number of active meds in group 2 & 2,412 & 2,693 & 2,783 & 2,700 & 2,157 & 1,354 \\
\hline Total number of active meds in group 3 & 14,787 & 20,179 & 26,874 & 34,387 & 43,583 & 57,225 \\
\hline Total number of active meds in group 4 & 17,960 & 23,643 & 30,323 & 37,682 & 46,208 & 58,904 \\
\hline Count of all CHF patients in group 1 & 250 & 256 & 217 & 194 & 158 & 112 \\
\hline Count of all CHF patients in group 2 & 329 & 371 & 379 & 368 & 295 & 131 \\
\hline Count of all CHF patients in group 3 & 663 & 868 & 1,112 & 1,407 & 1,715 & 2,024 \\
\hline Count of all CHF patients in group 4 & 1,242 & 1,495 & 1,708 & 1,969 & 2,169 & 2,317 \\
\hline
\end{tabular}

Figure 3 Continued.

Compliance Status Documentation, for which interprofessional discussions on the SKN system progressed through the full spectrum of themes from "problem statements" to "collective learning (aha) moments," to lay a foundation for practice change. These learning dynamics were associated with distinct improvements trends in both measures of MU of EHR MedRec. It would be relevant to note that we refrained from using broad outcome measures of "medication discrepancies" or "medication list accuracy," because of the lack of "truth" in what the accurate medication list was. This was further complicated by factors that can influence the "accuracy" of the list from the view of the provider on either end of a transition point, in regard to what medications the patient "should" be taking, and the 
view from the patient in regard to what they are actually taking. On the other hand, the two measures of MU of EHR MedRec utilized in this project, were most appropriate for our exploratory study, not only because they served as objective measures of MU of EHR MedRec technology, but also because they directly emerged from the interprofessional discussions on the SKN system, as having the greatest potential to reduce medication discrepancies and promote medication list accuracy for patients at AU Health.

In regard to the participants who made a difference on the SKN, results suggest that 3 of the 5 SKN Moderators (ie, $\mathrm{CMO}, \mathrm{CMIO}$, and the PI), played a crucial role in facilitating interprofessional knowledge exchange on issues related to EHR MedRec to promote collective learning of best practices. In both threads of discussion, initial "problem-solving statements" and "best-practice assertions" often emerged from among SKN Users or frontline providers, which provided a momentum for SKN Moderators to reinforce these types of messages. SKN Moderators also played key roles in providing "IT system education" and synthesizing lessons learned from Yammer discussions, to generate "collective learning (aha) moments," and create a foundation for practice change. All SKN Moderators also played an important role in the timely sharing of data and analytics related to both measures of MU of EHR MedRec on the SKN system, to enable data discussions and problem-solving. Additionally, although champions for change among SKN Users, often emerged from among pharmacists and nurses, physicians also actively participated in corroborating best-practice assertions and problem-solving statements, when they were presented with evidence linking practices to outcomes (which helped provide a rationale or the "why" for practice change).

\section{Implications for practice}

Results from this exploratory study suggest that an SKN system could be a valuable tool in enabling MU of EHR MedRec technology. From a broader perspective, SKN could be useful in enabling successful implementation of "complex innovations," ie, practice changes requiring coordination of care across the continuum, with an additional layer of complexity posed by health IT implementation (like MU of EHR MedRec technology).

In particular, results suggest that SKN helped achieve progress in MU of EHR MedRec technology, by addressing the implementation-challenges in the correct sequence, ie, by first enabling collective learning of the value of best practices in EHR MedRec, which, in turn, helped improve shared understanding of the EHR MedRec workflow at the system level. Our experience suggests that this type of collective learning of the "big picture," may be essential to have in place before embarking on any form of IT-training of practitioners to address socio-technical issues. Results also suggest that an SKN system may be a particularly valuable tool for institutions where practice change (eg, EHR Meaningful Use) needs to occur with existing resources, through workflow changes, as opposed to new resource outlays, eg, dedicated pharmacy techs in every unit. In this regard, one may argue that restricting the responsibility of EHR MedRec to dedicated resources might hinder the Meaningful Use of EHR technology if there are sudden changes to the financial situation, and dedicated resources are not sustainable anymore. In such situations, engaging all provider subgroups in developing a shared understanding of the value of EHR MedRec and the system-level EHR MedRec workflow, could be beneficial to any $\mathrm{HCO}$.

The above discussion provides insight into the following evidence-based management strategies for enabling successful implementation of complex innovations:

1. At the start of the effort, create a knowledge sharing and learning mechanism (eg, SKN) among a critical mass of providers, expected to implement practice changes. The mechanism should facilitate tacit knowledge exchange on issues experienced with current practices, as well as possible resolutions to those issues.

2. Conduct proactive, periodic communications (from senior leadership) on benefits of adhering to best practices and consequences of not adhering to them.

3. Create shared understanding of the value of best practices (ie, the link between practices and outcomes) or the answer to the question of "why" practices need to change, to gain provider engagement in changing practices, before embarking on IT-training of providers to address sociotechnical challenges.

4. Develop capacity to collect, analyze, and disseminate data on best-practice measures among providers to promote a scientific (research-based) approach to learning and improvement.

5. Enable champions for change to emerge from among providers, to voice the need for culture change for enabling successful best-practice implementation; and reinforce these messages with proactive, periodic communication from senior leadership. 
6. Create a "learning health system," by synthesizing lessons learned to facilitate "collective learning (aha) moments" across provider subgroups and care settings; and encourage providers to spread the learning within the broader institution. Management research has suggested framing practice issues as a "learning challenge" rather than a "performance challenge," and addressing them in the form of non-threatening pilot research projects, to engage providers in improvement. ${ }^{32,33}$ This project fulfilled both criteria to demonstrate meaningful results. This suggests that other health systems could also benefit from developing similar capabilities to become "learning health systems."

\section{Implications for theory}

Results and insights from this study serve to reinforce the theoretical framework that communication networks rich in brokerage and hierarchy, ie, proactive periodic top-down communication of best practices, may be effective in engaging professional subgroups to exchange tacit (practice-based) knowledge exchange, to foster collective learning and enable change in HCOs. Additionally, while this framework simply suggests that this type of communication structure can enable provider engagement, learning, and change, the results from this study, provide profound insights into how interprofessional learning occurs in an $\mathrm{HCO}$, to lay a foundation for practice change (MU of EHR MedRec technology), as summarized in Figure 2. While past studies have shed light on the factors enabling change implementation in HCOs ("the what"), there is limited understanding of how learning and change occur in HCOs ("the how"). ${ }^{33}$ By addressing this gap, this study makes a significant contribution to the theoretical literature on organizational learning and change implementation in HCOs.

\section{Limitations and future research avenues}

This study is limited in being restricted to one health system, and like any other implementation study, it is influenced by context in which the practice (EHR MedRec) is implemented at the institution. Correspondingly, the practice implications are also context sensitive. However, this may also be viewed as a strength of the study, in that, the exploratory and qualitative study design helped gain insight into dynamics of interprofessional learning, within the context of implementing new practices in a HCO, which helps to address a key gap in the literature on how learning and change occur in HCOs. Future research could help evaluate the generalizability of results from this exploratory study, through large-scale controlled experiments to investigate causal relationships between SKN Use and EHR Meaningful Use, across a wide variety of HCOs. Another future research avenue would be to examine the utility of an SKN system as a tool for enabling successful implementation of other complex innovations involving coordination of care and health IT implementation, like, for example, risk assessment for sepsis prevention.

\section{Conclusion}

Results of this exploratory study show that SKN Use was associated with Meaningful Use of EHR MedRec technology, at the study institution. The study suggests that an SKN system could be a valuable tool in enabling interprofessional knowledge exchange and learning to facilitate complex practice change (EHR Meaningful Use). Future large-scale studies of SKN use in HCOs could help to generate a systematic evidence-base of management strategies for promoting EHR Meaningful Use, which in turn, could be used to prompt federal EHR vendors to incorporate SKN features into EHR systems.

\section{IRB approval}

The study was approved by the IRB at Augusta University. IRB approval was obtained prior to data collection.

\section{Acknowledgment}

This study was supported by grant number R21HS024335 from the Agency for Healthcare Research and Quality (AHRQ)

\section{Disclosure}

The authors report no conflicts of interest in this work.

\section{References}

1. AHRQ, Agency for Healthcare Research and Quality. Patient safety primer: medication reconciliation; 2016. Available from: https://psnet. ahrq.gov/primers/primer/1/medication-reconciliation. Accessed October 15, 2017.

2. Barnsteiner JH. Medication Reconciliation. In: Hughes RG, editor Patient Safety and Quality: An Evidence-Based Handbook for Clinicians. Rockville, MD: Agency for Healthcare Research and Quality (US); Chapter 38. 2008. Available from: http://www.ncbi nlm.nih.gov/books/NBK2648/

3. CMS Centers for Medicare and Medicaid Services. Stage 2 eligible professional meaningful use core measures measure 14 of 17; 2012 Available from: https://www.cms.gov/Regulations-and-Guidance/ Legislation/EHRIncentivePrograms/downloads/Stage2_EPCore_14 MedicationReconciliation.pdf. Accessed November 1, 2016.

4. HealthIT.Gov. Achieving meaningful use stage 2: medication reconciliation; 2014. Available from: https://www.healthit.gov/providersprofessionals/achieve-meaningful-use/core-measures-2/medicationreconciliation. Accessed October 15, 2016 
5. Boockvar KS, Santos SL, Kushniruk A, Johnson C, Nebeker JR. Medication reconciliation: barriers and facilitators from the perspectives of physicians and pharmacists. J Hosp Med. 2011;6:329-337. doi:10.1002/jhm.891

6. Clay BJ, Halasyamani L, Stucky ER, Greenwald JL, Williams MV. Results of a medication reconciliation survey from the 2006 society of hospital medicine national meeting. J Hosp Med. 2008;3(6):465472. doi:10.1002/jhm.295

7. Rose AJ, Fischer SH, Pasche-Orlow MK. Beyond medication reconciliation: the correct medication list. J Am Med Assoc. 2017;317 (20):2057-2058. doi:10.1001/jama.2017.4628

8. Sanchez HS, Sadie SS, Santos SL, Boockvar K. Implementing medication reconciliation from the planner's perspective: a qualitative study. BMC Health Serv Res. 2014;14:290.

9. Greenwald JL, Halasyamani L, Greene J, et al. Making inpatient medication reconciliation patient centered, clinically relevant and implementable: a consensus statement on key principles and first steps. J Hosp Med. 2010;5(8):477-485. doi:10.1002/jhm.791

10. Van Sluisveld N, Zegers M, Natsch S, Wollersheim H. Medication reconciliation at hospital admission and discharge: insufficient knowledge, unclear task reallocation and lack of collaboration as major barriers to medication safety. BMC Health Serv Res. 2012;12:170. doi:10.1186/1472-6963-12-170

11. Grossman J, Gourevitch R, Cross DA. Hospital Experiences Using Electronic Health Records to Support Medication Reconciliation. Detroit, MI, USA: National Institute for Healthcare Reform (NIHCR) Research Brief Number 17; 2014.

12. Mueller SK, Sponsler KC, Kripalani S, Schnipper JL. Hospitalbased medication reconciliation practices: a systematic review. Arch Intern Med. 2012;172:1057-1069. doi:10.1001/archinternm ed.2012.2246

13. Porcelli PJ, Wlatman LR, Brown SH. A review of medication reconciliation issues and experiences with clinical staff and information systems. Appl Clin Inform. 2010;1:442-461. doi:10.4338/ACI-2010-01-RA-0005

14. Rangachari P. Overcoming the unintended adverse consequences of implementing health IT through human resource and knowledge management. In: Blair JD, Fottler MD, Savage GT, editors. Advances in Health Care Management. Vol. 9. Bingley, UK: Emerald; 2010a:163-194.

15. Smith P. Med wreck: proposing a solution for the nightmare of medication reconciliation; Ebook; 2017. Available from: https://healthitaccelerator. com/med-wreck/ Accessed October 16, 2017.

16. AHRQ, Agency for Healthcare Research and Quality. Using Social Knowledge Networking (SKN) technology to enable meaningful use of EHR technology; 2016. Available from: https://healthit.ahrq.gov/ ahrq-funded-projects/using-social-knowledge-networking-skn-tech nology-enable-meaningful-use-ehr. Accessed June 15, 2017

17. Rangachari P. Implementing a Social Knowledge Networking (SKN) system to enable meaningful use of an EHR medication reconciliation system: a study protocol. Risk Manag Healthc Policy. 2018;11:45-53. doi:10.2147/RMHP.S152313
18. Plsek P. Redesigning health care with insights from the science of complex adaptive systems (Appendix B, 309Y322). In: Institute of Medicine, Crossing the Quality Chasm. Washington, DC, USA: National Academies Press; 2001.

19. Anderson A, McDaniel R. Managing health care organizations: where professionalism meets complexity science. Health Care Manage Rev. 2000;25(1):83-92.

20. Burt R. Network structure of social capital. In: Sutton RI, Staw BM, editors. Research in Organizational Behavior. Vol. 22. Greenwich, CT: JAI Press; 2000.

21. Hansen MT. The search-transfer problem: role of weak ties in sharing knowledge across organizational units. Adm Sci Q. 1999;44(1):82111. doi: $10.2307 / 2667032$

22. Rangachari P. Knowledge sharing networks related to hospital quality measurement and reporting. Health Care Manage Rev. 2008;33 (3):253-263. doi:10.1097/01.HMR.0000324910.26896.91

23. Rangachari P. Knowledge sharing networks in professional complex systems. J Knowl Manag. 2009;13(3):132-145. doi:10.1108/ 13673270910962923

24. Rangachari P. Knowledge sharing and organizational learning in the context of hospital infection prevention. Qual Manag Health Care. 2010b;19(1):34-45. doi:10.1097/QMH.0b013e3181ccbd1d

25. Rangachari P. Using social knowledge networking technology to enable meaningful use of electronic health record technology in hospitals and health systems: A review article. J Hosp Adm. 2014;3 (6):66-78. doi:10.5430/jha.v3n6p66

26. Rangachari P, Madaio M, Rethemeyer RK, et al. The evolution of knowledge exchanges enabling successful practice change in two intensive care units. Health Care Manage Rev. 2015a;40(1):65-78. doi:10.1097/HMR.0000000000000001

27. Rangachari P, Madaio M, Rethemeyer RK, et al. Cumulative impact of periodic top-down quality improvement communications on infection prevention practices and outcomes in two units. Health Care Manage Rev. 2015b;40(4):324-336. doi:10.1097/ HMR.0000000000000038

28. Gabriel MH, Swain M. "E-Prescribing Trends in the United States" (PDF). ONC Data Brief. Office of the National Coordinator for Health IT; 2014. September 2016

29. Richards L. Using NVivo in Qualitative Research. London: Sage; 1999. ISBN 0-7619-6525-4.

30. Braun V, Clarke V. Using thematic analysis in psychology. Qual Res Psychol. 2006;3(2):77-101. doi:10.1191/1478088706qp063oa

31. Elo S, Kyngas $\mathrm{H}$. The qualitative content analysis process. J Adv Nurs. 2007;62(1):107Y115.

32. Nembhard IM, Alexander JA, Hoff TJ, Ramanujam R. Why does the quality of health care continue to lag? Insights from management research. Acad Manag Perspect. 2009;23(1):24-41. doi:10.5465/ AMP.2009.37008001

33. Rangachari P. Innovation implementation in the context of hospital QI: lessons learned and strategies for success. Innov Entrep Health. 2018b;5:1-14. doi:10.2147/IEH.S151040 


\section{Publish your work in this journal}

The Journal of Healthcare Leadership is an international, peer-reviewed, open access journal focusing on leadership for the health profession. The journal is committed to the rapid publication of research focusing on but not limited to: Healthcare policy and law;Theoretical and practical aspects healthcare delivery; Interactions between healthcare and society and evidence-based practices; Interdisciplinary decision-making;
Philosophical and ethical issues; Hazard management; Research and opinion for health leadership; Leadership assessment. The manuscript management system is completely online and includes a very quick and fair peer-review system. Visit http://www.dovepress.com/ testimonials.php to read real quotes from published authors.

Submit your manuscript here: https://www.dovepress.com/journal-of-healthcare-leadership-journal 